\title{
The statistics of foreshock cavities: results of a Cluster survey
}

\author{
L. Billingham ${ }^{1}$, S. J. Schwartz ${ }^{1}$, and D. G. Sibeck ${ }^{2}$ \\ ${ }^{1}$ Space and Atmospheric Physics, Blackett Laboratory, Imperial College London, London, UK \\ ${ }^{2}$ Goddard Space Flight Center, Greenbelt, MD, USA
}

Received: 3 April 2008 - Revised: 7 October 2008 - Accepted: 7 October 2008 - Published: 20 November 2008

\begin{abstract}
We use Cluster magnetic field, thermal ion, and energetic particle observations upstream of the Earth's bow shock to investigate the occurrence patterns of foreshock cavities. Such cavities are thought to form when bundles of magnetic field connect to the quasi-parallel bow shock. Shockprocessed suprathermal ions can then stream along the field, back against the flow of the solar wind. These suprathermals enhance the pressure on shock-connected field lines causing them to expand into the surrounding ambient solar wind plasma. Foreshock cavities exhibit depressions in magnetic field magnitude and thermal ion density, associated with enhanced fluxes of energetic ions. We find typical cavity duration to be few minutes with interior densities and magnetic field magnitudes dropping to $\sim 60 \%$ of those in the surrounding solar wind. Cavities are found to occur preferentially in fast, moderate magnetic field strength solar wind streams. Cavities are observed in all parts of the Cluster orbit upstream of the bow shock. When localised in a coordinate system organised by the underlying physical processes in the foreshock, there is a systematic change in foreshock cavity location with IMF cone angle. At low (high) cone angles foreshock cavities are observed outside (inside) the expected upstream boundary of the intermediate ion foreshock.
\end{abstract}

Keywords. Interplanetary physics (Discontinuities; Energetic particles; Planetary bow shocks) - Space plasma physics (Shock waves)

\section{Introduction}

Kinetic processes occurring upstream of the terrestrial bow shock can greatly perturb the parameters of the solar wind incident on the dayside magnetosphere. The region upstream of the bow shock contains many phenomena: Field aligned

Correspondence to: L. Billingham

(laurence.billingham@imperial.ac.uk) ion beams form when a fraction of the solar wind meeting the bow shock is reflected (Sonnerup, 1969), or by upstream leakage of magnetosheath ions (Edmiston et al., 1982). ULF waves are formed as a result of instabilities between the solar wind beam and the field aligned population; they are observed (Greenstadt et al., 1968; Fairfield, 1969), often associated with crescent shaped intermediate ion distributions (Fuselier et al., 1986), some way downstream of the field aligned beams. Models of the foreshock (Skadron et al., 1986) have shown that Earth-incident solar wind is slowed and deflected by the pressure gradient induced by the presence of the foreshock. The deflection and deceleration are accompanied by significant perturbations in density and magnetic field. Local hybrid simulations of the interaction between the solar wind and a spatially limited counterstreaming beam (Thomas and Brecht, 1988) develop crater like structures of depressed density and magnetic field bounded by enhanced edges, that may likewise perturb the bulk solar wind flow.

Events matching these predictions have been found in spacecraft data sets. Hot Flow Anomalies (HFAs) (Schwartz et al., 1985; Thomsen et al., 1986; Paschmann et al., 1988) exhibit large flow deflections and temperature enhancements. Interactions between a subset of solar wind discontinuities (Schwartz et al., 2000b) and the bow shock have been found to explain HFA signatures better than the Thomas and Brecht (1988) model.

Foreshock cavities, first reported by Wibberenz et al. (1985) as crater-like magnetic field dropouts filled with energetic ions in ISEE 2 data (see also Fairfield et al., 1990; Sibeck et al., 2002), may be a better match for the predictions of Thomas and Brecht (1988). Cavities are thought to form when magnetic field bundles connect to the quasiparallel bow shock. This allows suprathermal ions to stream from the shock along the field and back against the bulk flow the solar wind (Schwartz et al., 2006). Enhanced the pressures on shock-connected field lines, due to the additional

Published by Copernicus Publications on behalf of the European Geosciences Union. 
suprathermal population, drives expansion into the surrounding solar wind plasma.

Case studies of foreshock cavities (e.g. Sibeck et al., 2002; Schwartz et al., 2006; Sibeck et al., 2004) have shown that, unlike HFAs, they need not be associated with interplanetary discontinuities. There is little heating or deflection of the solar wind bulk flow within these case study events, but a population of suprathermal ions is present in addition to the solar wind population.

Foreshock cavities and Hot Flow Anomalies are observed to have a measurable impact on the dynamics of the magnetosphere. Fairfield et al. (1990), using AMPTE and GOES data, found that correlated density and field perturbations, due to local solar wind bow shock interaction, were responsible for compressions of the magnetosphere. Sibeck et al. (2000) presented a case study in which it was possible to identify a causal chain for transient magnetopause motion observed at geosynchronous orbit, tracking pressure variations through the magnetosheath, to their origin: changes in the solar wind magnetic field interacting with the bow shock excavating cavities. Murr and Hughes (2003) were able to identify foreshock cavities as the triggers of several travelling convection vortices in the ionosphere.

A previous survey of foreshock cavities was carried out by Sibeck et al. (2001) using IMP-8 data. In seven months of observations, they identified 292 foreshock cavities, finding that they occurred preferentially the pre-noon sector, and in solar wind streams with fast bulk flow speeds. Central magnetic fields were found to be depressed by $20 \%$ with respect to solar wind values. Low time resolution plasma data prevented Sibeck et al. (2001) from investigating the statistics of the densities and velocities in cavity interiors. Although magnetic depressions of a factor of two had been found in previous case studies (e.g. Sibeck et al., 1989), the relatively weak cavity signatures in the more recent survey were attributed to the greater distance of the IMP- 8 orbit from the bow shock.

Recently, events have been observed upstream of the Martian (Øieroset et al., 2001) and Kronian (Masters et al., 2008) bow shocks that share many of the characteristics of terrestrial HFAs and foreshock cavities. This suggests that cavities due to local shock interactions occur in a variety of plasma regimes and that they may be as ubiquitous a phenomenon as collisionless shocks themselves.

This paper presents the results of a survey of foreshock cavities seen by Cluster close to the bow shock. We present the first survey of thermal ion distribution parameters inside foreshock cavities. Studies of individual cavities (e.g. Schwartz et al., 2006) have shown that they need not form along underlying interplanetary discontinuities; we investigate the nature of the changes in plasma conditions across a large set of cavities to determine if this is generally applicable.

The magnetic field and density profiles found by Skadron et al. (1986) for the traversal of a model foreshock resemble those observed for foreshock cavities. It has been suggested (e.g. by Sibeck et al., 2008, in light of recent global hybrid simulation results) that foreshock cavities might be transient encounters with the moving ion foreshock. Previous authors (e.g. Schwartz et al., 2006) model foreshock cavities as being due to pre-existing fine scale structure in the ambient solar wind temporarily altering the local magnetic connection to the bow shock. The Sibeck et al. (2008) suggestion may be distinguished from this scenario, as transient encounters with the foreshock boundary must cluster around the equilibrium position of the foreshock edge. We test the Sibeck et al. (2008) boundary encounter model by mapping the locations of cavities against boundaries of the contiguous ion foreshock.

\section{Dataset and selection criteria}

Our survey covers the 2005 and 2006 Cluster dayside seasons; the periods between 15 December 2004 and 26 May 2006 when the Cluster spacecraft were upstream of the Earth's bow shock. We draw on four second resolution data from: the FluxGate Magnetometer (FGM, Balogh et al., 2001), moments of the thermal $\left(5 \mathrm{eV} \mathrm{e}^{-1}\right.$ to $\left.32 \mathrm{keV} \mathrm{e}^{-1}\right)$ ion distribution from the Hot Ion Analyser part of the Cluster Ion Spectrometer (CIS(HIA), Rème et al., 2001), and high energy ( $\geq 27 \mathrm{keV}$ ) ion fluxes from RAPID (Wilken et al., 2001). Events were selected as foreshock cavities by requiring suprathermal ion fluxes enhanced over background levels along with concurrent depressions in magnetic field magnitude and thermal ion density. Another class of kinetic event near the earth's bow shock, Hot Flow Anomalies (HFA) may present similar profiles to foreshock cavities. We distinguished between HFAs and foreshock cavities by rejecting events showing marked increases in CIS(HIA) temperature or where interior bulk flow speeds deviated by $\geq 20 \%$ from the ambient solar wind speed.

A previous survey of foreshock cavities was carried out by Sibeck et al. (2001) using data taken by the IMP-8 spacecraft in January through August 1995. Some differences in the methodology of that work and the present study should be kept in mind when results are compared. Our selection criteria are more stringent than those of Sibeck et al. (2001) who required only that energetic particle fluxes exceed a given threshold. In light of this, we expect to identify events as foreshock cavities at a lower rate. The orbit of the IMP-8 spacecraft lay approximately in the ecliptic plane, and had an apogee of over $40 R_{E}$ during the Sibeck et al. (2001) survey; much further from Earth than Cluster. The near polar Cluster orbit has an of apogee of $19.6 R_{E}$, the orbit tends to cross the bow shock, at grazing angles, some way downstream of the shock's nose. Shock proximity combined with the out of ecliptic orbit allows the Interplanetary Magnetic Field (IMF) to intersect the shock over a greater range of angles at Cluster than at IMP-8. Both survey periods fall in the late declining 
phase of the solar cycle, so we expect the solar wind streams (Corotating Interaction Regions etc.) encountered during the course of each study to be broadly similar.

Averages of pre- and post-foreshock cavity conditions were typically taken over a two minute window. The averaging period was extended if conditions were especially disturbed, and reduced if other events/discontinuities prevented the use of the full two minutes of data. Averaging intervals were placed as close as possible to each foreshock cavity without including either the central depressions or any edge enhancements. Values presented for cavity interiors are those measured coincident with the minimum of the magnetic field magnitude in the four second resolution data. As conditions within cavities are often highly disturbed the exact timing and depth of the field minimum could be expected to change for different sampling rates/averaging schemes (the minimum of the "trough" tending to be lower for higher cadence time series).

To identify an event as a foreshock cavity, we must be able to discriminate it from its surroundings. Any cavities embedded in a ULF wave field or in regions of significant electromagnetic turbulence, for example, could not be picked out as unique events. Foreshock cavity interiors are generally highly disturbed, consistent with quasi-parallel connection to the bow shock; however, their surroundings must be relatively quiescent, in order for them to be identified. This criterion is consistent with previously reported examples of foreshock cavities; however, it limits our ability to detect any events that might occur in the turbulent regions downstream of field aligned ion beam foreshock.

We put the plasma environment in which foreshock cavities are embedded in context by comparing it to average data from each hour when Cluster was upstream of the bow shock during the survey period. The cavities themselves are included in these intervals, but the hourly averaging suppresses their influence on the reported parameters. Compared to the few minute averaging window used for the immediate cavity surroundings, data averaged over an hourly window would be expected to show considerably less variability.

\section{An example cavity}

Figure 1 gives an overview of field and plasma conditions during an event centred around 14:15 (UT) on 28 December 2005 when Cluster was upstream of the southern dusk bow shock. Corresponding depressions in magnetic field magnitude (heavy black line in the first panel) and in thermal ion density (second panel), along with elevated fluxes of suprathermal ions (third panel) mark this event as probable a foreshock cavity. The lack of significant change in the bulk flow speed (third panel) or heating of the solar wind ions (bottom panel) confirms this event as a foreshock cavity rather than an HFA. The magnetic field observed in the centre of the cavity is deflected by $\sim 80^{\circ}$ from the pre-

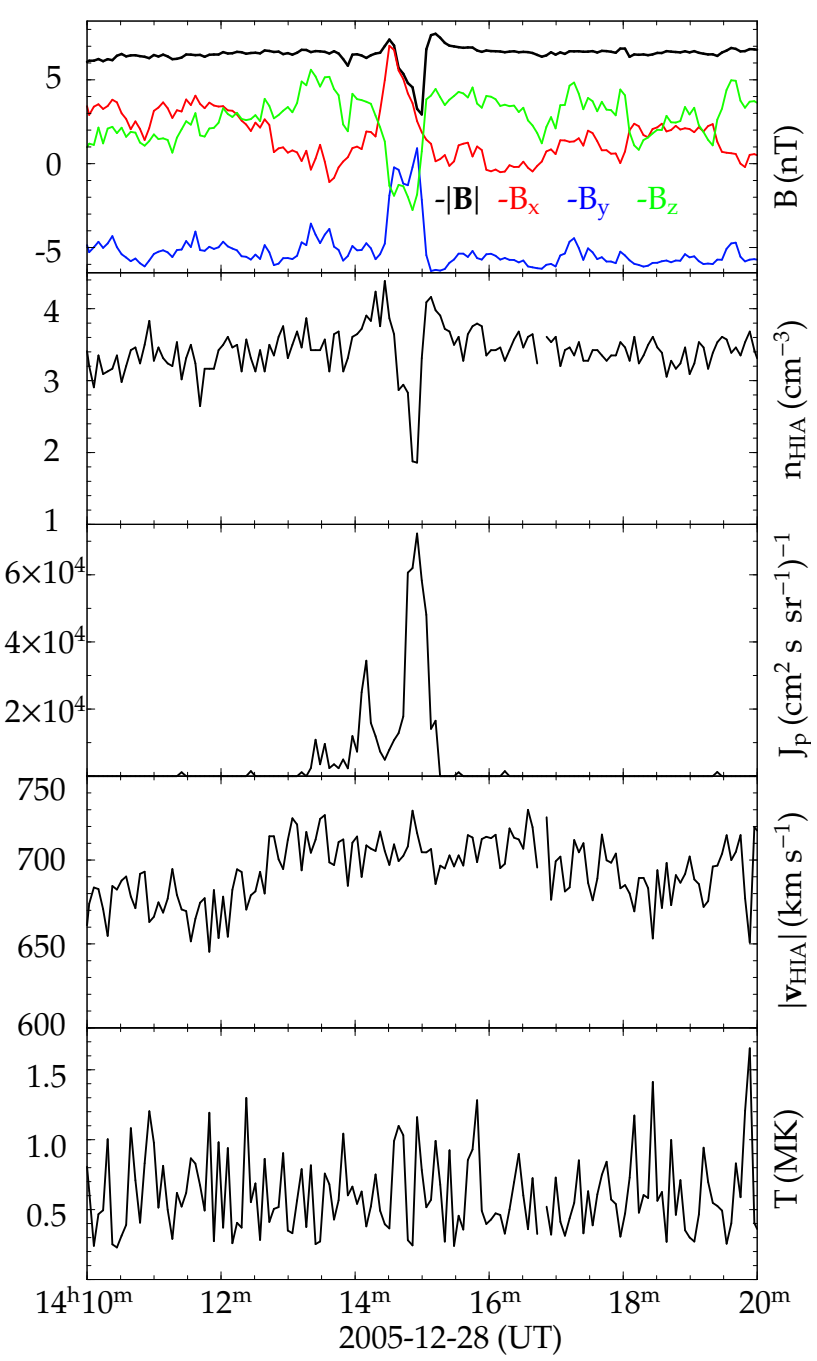

Fig. 1. Stacked time series traces giving an overview of the foreshock cavity observed by Cluster on 28 December 2005, 14:15:00 when the spacecraft were at $(8.2,16.1-6.0) R_{E}$ GSE. All parameters were measured by Cluster 3 . The top panel shows the magnitude of the magnetic field and its components in GSE coordinates. The second panel shows the density of the thermal ion population from CIS moments. The third panel shows the flux of energetic $\left(\geq 27 \mathrm{keV} \mathrm{e}^{-1}\right)$ ions measured by the RAPID instrument. The bottom two panels show the bulk flow speed and temperature of the ions measured by CIS. These signatures are typical of foreshock cavities with correlated dropouts in field strength and density lasting $50 \mathrm{~s}$, associated with enhanced suprathermal fluxes. The central field and density both drop to 0.59 of their exterior values. The preand post-event fields make an angle of $12^{\circ}$, the event has not formed on a significant solar wind discontinuity. This, along with the lack of significant changes in ion velocity and temperature, mark this event as a foreshock cavity rather than a HFA.

and post-event fields which point in almost the same direction. The components of the field in the $y_{\mathrm{GSE}}$ (dusk-dawn) and $z_{\mathrm{GSE}}$ (north-south) directions fall inside the cavity with 


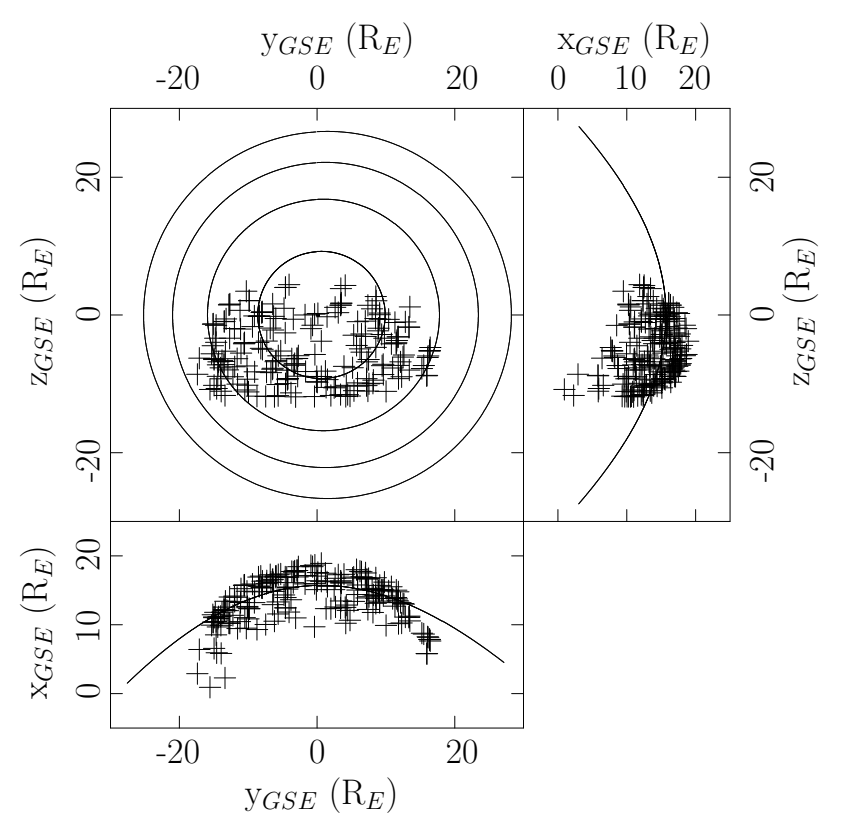

Fig. 2. Locations of the 204 foreshock cavities identified during the 2005 and 2006 Cluster dayside seasons. The left hand part shows the cavities projected onto the yz-, xy-, and xz-GSE planes. Sections through model bow shock are also included; the concentric curves in the yz-plane are sections taken through the model at the terminator and at 4, 8, and $12 R_{E}$ sunward of the Earth.

respect to ambient. The remaining field component parallel to the Earth-Sun line favours magnetic connection to the bow shock. This allows suprathermal ions to stream away from the bow shock, along the field, and reach the spacecraft. There are clearly some suprathermthermals observed outside the central magnetic depression, behaviour previously noted by Schwartz et al. (2006) and Sibeck et al. (2001). These ions, which have energies $\geq 27 \mathrm{keV}$, suggest that the plasma immediately before the field depression is connected to the terrestrial bow shock. However the exterior field is not significantly turbulent and there are no coherent waves. The cavity is not embedded within the well developed ULF foreshock, but there is a burst of energetic ions, associated with a small field rotation just before the cavity at $\sim 14: 12: 30$ which is probably a beam aligned to the pre-cavity field. If this is the case, it implies that this pre-cavity field connects to the bow shock, and that we are downstream of the tangent fieldline.

\section{Foreshock cavity locations}

\subsection{Comparison with Cluster orbit}

During the survey period, Cluster was upstream of the bow shock for $\sim 270$ days and observed 204 events that we iden- tify as foreshock cavities. This gives an occurrence rate of $\sim 0.75$ foreshock cavities per day, almost two per Cluster orbit. This is less than the 1.2 per day reported by Sibeck et al. (2001), in line with our expectations (see Sect. 2).

We next determine if there is any preferred location to observe foreshock cavities. Figure 2 shows the location of each observed foreshock cavity projected onto the xy-, yz, and xz-planes of the GSE coordinate system. For reference we also plot the model bow shock of Slavin and Holzer (1981). Most cavities are observed slightly south of the ecliptic, within $15 R_{E}$ of the noon-midnight meridian and in a broad band between 5 and $18 R_{E}$ sunward of the terminator. Figure 3 plots histograms of the locations of foreshock cavities projected and binned along each GSE axis. Also shown are histograms of the Cluster orbit corresponding to solar wind conditions.

Cavities were found throughout the parts of the Cluster orbit outside of the bow shock, out to apogee at $19.6 R_{E}$. There is a slight asymmetry in the distribution of locations along the $y$ axis, with $\sim 55 \%$ of the cavities observed in the pre-noon $(y<0)$ sector. Sibeck et al. (2001) also report preferentially observing cavities in the pre-noon sector. However the bias they find, and attribute to the prevalence of spiral IMF, is much more marked than the slight asymmetry in our set of events. As Cluster remains much closer to the bow shock than IMP-8, a much broader range of IMF orientations connect it to the shock. For each of the three position components, the distribution of cavity locations is similar to the distribution of the orbit.

There is an apparent lack of cavities observed near the subsolar point, visible in both the yz panel of the three-axis projection and in the bins near the zero in the $y_{\mathrm{GSE}}$ histogram. Although this lack of cavities near the nose of the bow shock is visually striking, especially in Fig. 2, tests on the goodness of fit between the Cluster orbit and observed cavity locations (in each of the three GSE components) suggest that the distributions are only marginally different in a statistical sense.

Cluster is furthest upstream of the bow shock around its nose. As Sibeck et al. (2004) established that foreshock cavities become weaker farther from the shock, cavities observed by Cluster near the nose might be expected to be weaker than those found near the flanks. However, for this to fully explain the lack of cavities near the nose, cavity strength would have to fall off rapidly to below a threshold of detectability within the 19.6 $R_{E}$ Cluster orbit. As cavities have been observed at almost $40 R_{E}$, we conclude that increasing distance from the shock cannot be a major contributor to the lack of cavities near the sub-solar point.

\subsection{Comparison with foreshock location}

Recent global hybrid simulations (Sibeck et al., 2008) suggest that transient encounters with the leading edge of the foreshock (as field aligned ion-beams respond to changes in the solar wind magnetic field by moving back and forth over 

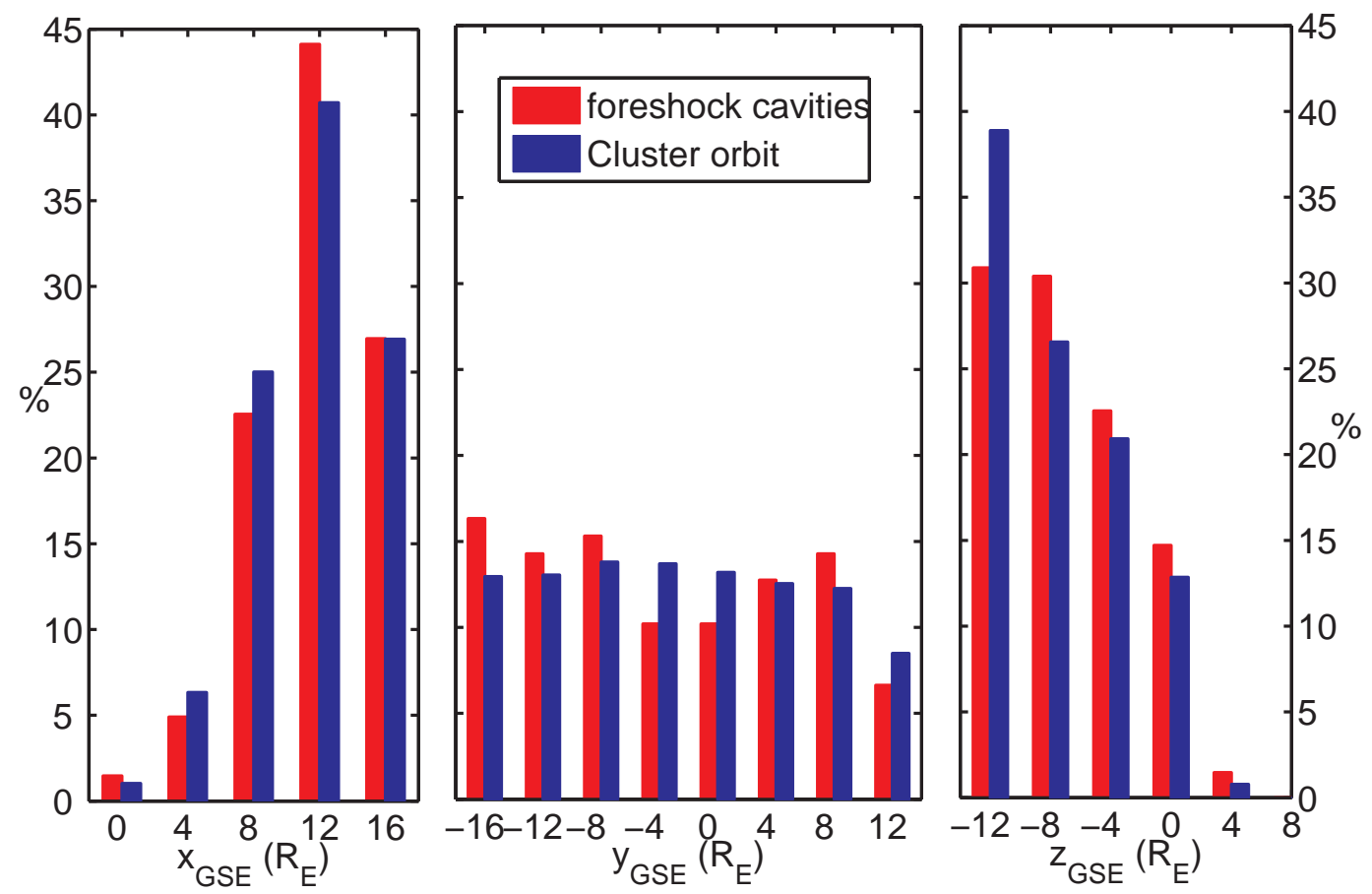

Fig. 3. Histograms of cavity locations projected onto each GSE axis along with hourly averages of the part of the Cluster orbit upstream of the bow shock. In each component, the distributions are similar. Foreshock cavities are observed throughout the Cluster orbit. More cavities are observed outside the pre-noon $\left(y_{\mathrm{GSE}} \leq 0\right)$ bow shock than post dawn; this asymmetry is most likely due to the IMF assuming a spiral orientation.

a spacecraft) may account for the foreshock cavity signatures observed in spacecraft time series.

Identification of foreshock cavities requires relatively quiescent background plasma conditions, like those often found in the unperturbed solar wind. Any cavity occurring within the fully developed ion foreshock could not be picked out as a discrete event. Thus, if cavities are transient encounters with the edge of the foreshock, it must move over the spacecraft in such a way as to leave relatively quiet conditions on either side; for example advancing over a spacecraft in the solar wind before retreating.

We have transformed our set of cavity observation points into a coordinate system organised by foreshock morphology, allowing the comparison of foreshock cavity locations with foreshock structures. The Solar Foreshock Coordinate (SFC) system was developed by Greenstadt and Baum (1986) to map the onset of ULF waves in the foreshock and has been used by Meziane and d'Uston (1998) to locate the edge of the intermediate ion foreshock.

Figure 4 explains how foreshock observations are organised by the SFC system. A section is taken through the bow shock in a plane containing the IMF vector and the solar wind velocity. In this plane, an event may be located by the coordinates $X_{F}$ and $D_{B T} . D_{B T}$ is the distance along the tangent field line from the point of tangency to a point immediately upstream of the event. $X_{F}$ is the distance, along the solar wind flow, from that point to the event. Shock-processed particles stream along the magnetic field, but also drift in the solar wind motional electric field; their trajectories form straight lines in SFC. Particles of a given energy can only access regions downstream of some bounding line. For energetic electrons this boundary is very near the tangent field line, slower ions would only be observed well Earthward of the tangent line.

Following Greenstadt and Baum (1986) and Meziane and d'Uston (1998), we approximate the direction of the solar wind velocity as parallel to the Earth-Sun line. We take the best-fit model of Slavin and Holzer (1981) to approximate position of the bow shock, and use an average of the magnetic fields observed immediately before and after each foreshock cavity. Although the shock model captures the typical position of the bow shock averaged over many shock crossings, the error in the location of the shock for any given event is considerable, leading to scatter in plots of observed $\left(D_{B T}, X_{F}\right)$. As the Slavin and Holzer (1981) model is a hyperboloid, it is not a good fit to the bow shock shape at the flanks. Errors in shock location increase dramatically for large $\left(D_{B T}, X_{F}\right)$. Accounting for this, we removed foreshock cavities having $X_{F}>30 R_{E}$, reducing our set of cavities from 204 to 145 . Cavities observed with large $X_{F}$ 


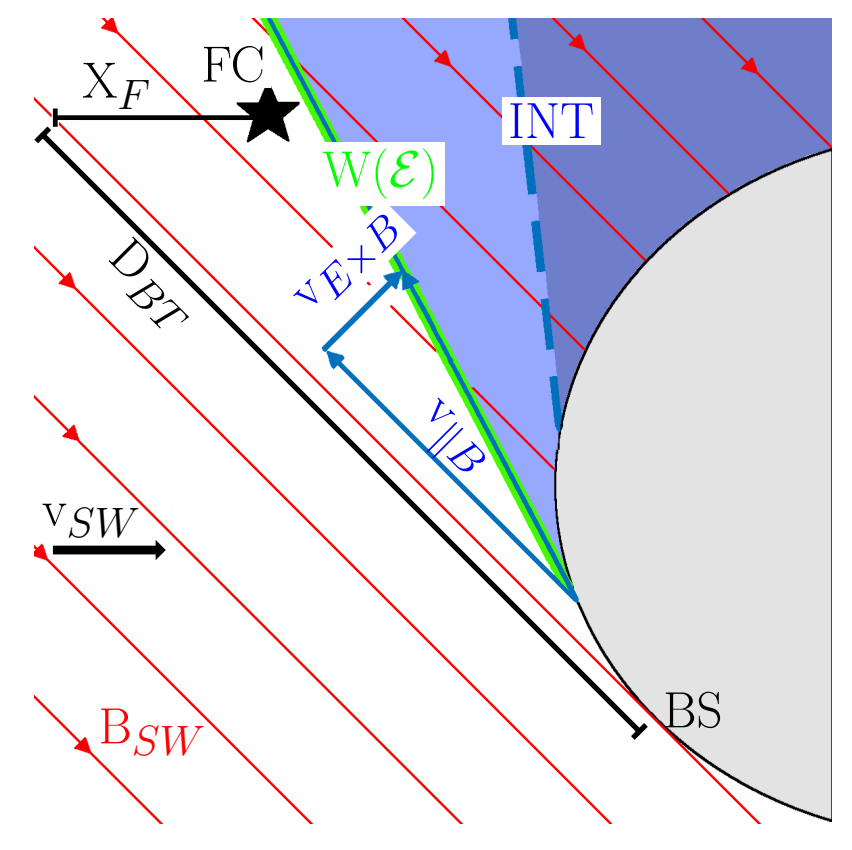

Fig. 4. A section through the bow shock (marked BS) in a plane containing the IMF vector and the solar wind flow $\left(B_{S W}\right.$ and $\left.v_{S W}\right)$. $D_{B T}$ is the distance along the tangent field line from the point of tangency to a point immediately upstream of the observation point. $X_{F}$ is the distance, in the direction of the solar wind flow, from that upstream point to the event location. In this plane, an event (here a foreshock cavity labelled FC) may be located by the coordinates $X_{F}$ and $D_{B T}$. It is only possible for particles of a given energy to access regions downstream of the boundary line marked $W(\mathcal{E})$ as they are subjected to $\boldsymbol{E} \times \boldsymbol{B}$ drift in the solar wind motional electric field (see Schwartz et al., 2000a). $W(\mathcal{E})$ is the upstream edge of the ion beam foreshock. This and other boundaries of the ion-foreshock (e.g. the intermediate foreshock boundary of Meziane and d'Uston, 1998, labelled INT) lie downstream of the tangent field line.

distances correspond to those occurring under nearly radial IMF conditions. Over a quarter of our set of 204 cavities occur for cone angles of $35^{\circ}$ or less. For low cone angle solar wind magnetic fields the intersection between field and the bow shock moves slowly along the shock surface allowing the long connection times discussed by Schwartz et al. (2006).

The orbit of the Cluster spacecraft imposes a selection effect in the SFC system. The orbit crosses bow shock relatively near the nose, but offset some distance from the subsolar point; this means that regions where $X_{F}$ is large cannot be sampled under high IMF cone angle conditions.

Figure 5 is a scatter plot of the location of each foreshock cavity in the SFC system. Thirty cavities were observed upstream of the nominal tangent field line; the plasma embedding these cavities is not connected to the nominal bow shock at all. If these cavities are encounters with the edge of the fully developed foreshock, this implies the edge must, on oc-

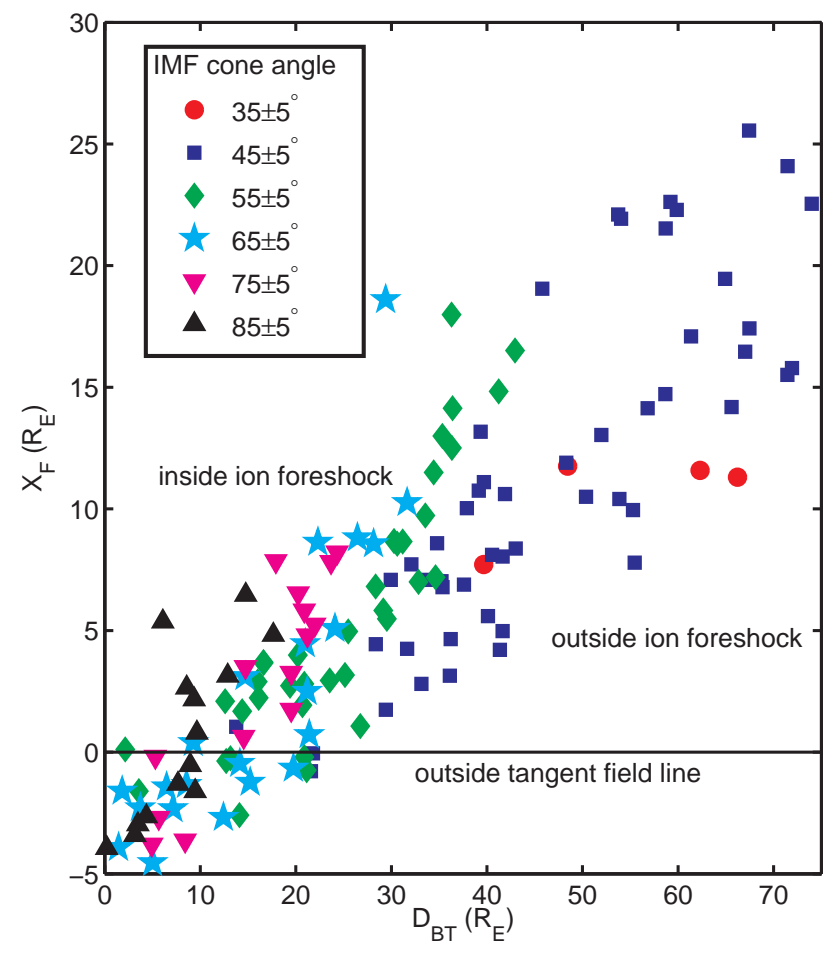

Fig. 5. Scatter plot of the location of foreshock cavities in the Solar Foreshock Coordinate system (see Fig. 4). Solid symbols show cavity observation locations. Events have been sorted by the cone angle between the IMF and the GSE x-axis; bins are indicated by colour. The $X_{F}=0$ line corresponds to the tangent field line. Events having $X_{F}<0$ were observed upstream of the field line tangentially intersecting the nominal bow shock. For other foreshock boundaries, studies (Greenstadt et al., 1968; Meziane and d'Uston, 1998) have not found a single line of demarcation that applies over a variety of upstream conditions. However, Fig. 6 compares the location of some foreshock cavities to appropriate foreshock boundary fits. Note that the upper-left region in these figures corresponds to positions deep within the ion foreshock, whilst the lower-right part maps to regions upstream of the foreshock.

casion, move at least $\sim 5 R_{E}$ sunward of its expected position.

A least squares fit to the cavity locations indicates there is an approximately linear correlation between $X_{F}$ and $D_{B T}$. This is the same behaviour that would be expected for a set of ion foreshock boundary encounters, since the trajectory of a suprathermal backstreaming ion, accounting for $\boldsymbol{E} \times \boldsymbol{B}$ advection, is a line in the SFC system (see the line labelled W in Fig. 4).

There is a systematic variation in cavity locations with the cone angle $\left(\theta_{B X}=\arccos \left(B_{x} /|\boldsymbol{B}|\right)\right)$ between the IMF vector and the Sun-Earth line. We bin our cavity observations by cone angle; each bin is $10^{\circ}$ wide, with centres at $\left\langle\theta_{B X}\right\rangle:=\left\{35^{\circ}, 45^{\circ}, 55^{\circ}, 65^{\circ}, 75^{\circ}, 85^{\circ}\right\}$, and contain $4,53,37,22,15$, and 14 cavities respectively. These bins are 
the same as those employed by Meziane and d'Uston (1998) in their study of the upstream boundary of the intermediate ion foreshock boundary. Intermediate ion distributions are observed, along with ULF waves, some way downstream of the field aligned beams that mark the ion-foreshock's leading edge (Hoppe and Russell, 1983; Eastwood et al., 2005). Figure 4 shows a sketch of this edge in relation to other foreshock boundaries.

As IMF cone angle increases, foreshock cavities are observed further downstream of ion foreshock edge; this can be deduced from the steepening of gradient of the sets of points in Fig. 5 with cone angle. This variation is more pronounced than, and in the opposite sense to, that reported for the intermediate ion foreshock boundary: see the lines for $\theta_{B x}=\left\{45^{\circ}, 75^{\circ}\right\}$ in Fig. 6 below and in Table 1 of Meziane and d'Uston (1998).

Figure 6 directly compares the observed locations of foreshock cavities and reported fits to the intermediate ion foreshock for large and small IMF cone angles. In the low cone angle limit, we show cavities having $\theta_{B X}=45^{\circ} \pm 5^{\circ}$ and compare their positions to the upstream intermediate ion foreshock boundary published by Meziane and d'Uston (1998) for the same range of cone angles (although we observe some cavities with $\theta_{B X}<40^{\circ}$, after imposing our $X_{F}<30 R_{E}$ criterion, they are too scarce for us to make meaningful comparisons with other studies). As IMF cone angle tends towards $90^{\circ}, \theta_{B X}$ bin occupancy drops again. Noting the similarity of the intermediate foreshock bounding lines for $75^{\circ}$ and $85^{\circ}$ (their gradients and intercepts are the same to within the reported errors), we conflate the foreshock cavity observations for our two highest cone angle bins; effectively rebinning them for $\theta_{B X}=80^{\circ} \pm 10^{\circ}$.

In Fig. 6, for each cone angle bin, the region in which foreshock cavities are observed is systematically offset from the line of the expected intermediate ion foreshock boundary. This behaviour is different to that expected for a series of foreshock edge encounters, which would be scattered around the boundary line.

The region downstream of the intermediate ion foreshock edge is characterised by significant turbulence. The large magnetic and density fluctuations would tend mask the signatures of any foreshock cavities embedded in this region. As cavities would be difficult to distinguish from the background plasma, we might expect to observe cavities preferentially in quiescent regions, upstream of the intermediate ion foreshock. When the IMF cone angle is low $\left(\theta_{B X} \sim 45^{\circ}\right)$ cavities are observed almost exclusively upstream of the nominal intermediate ion foreshock boundary. However, when the IMF cone angle is large $\left(\theta_{B X} \geq 70^{\circ}\right)$, foreshock cavities are found further downstream. The majority of these cavities lie downstream (above the lines in Fig. 6) of the nominal intermediate ion foreshock, but were nevertheless identifiable as individual, transient, events against a comparatively undisturbed background plasma environment.

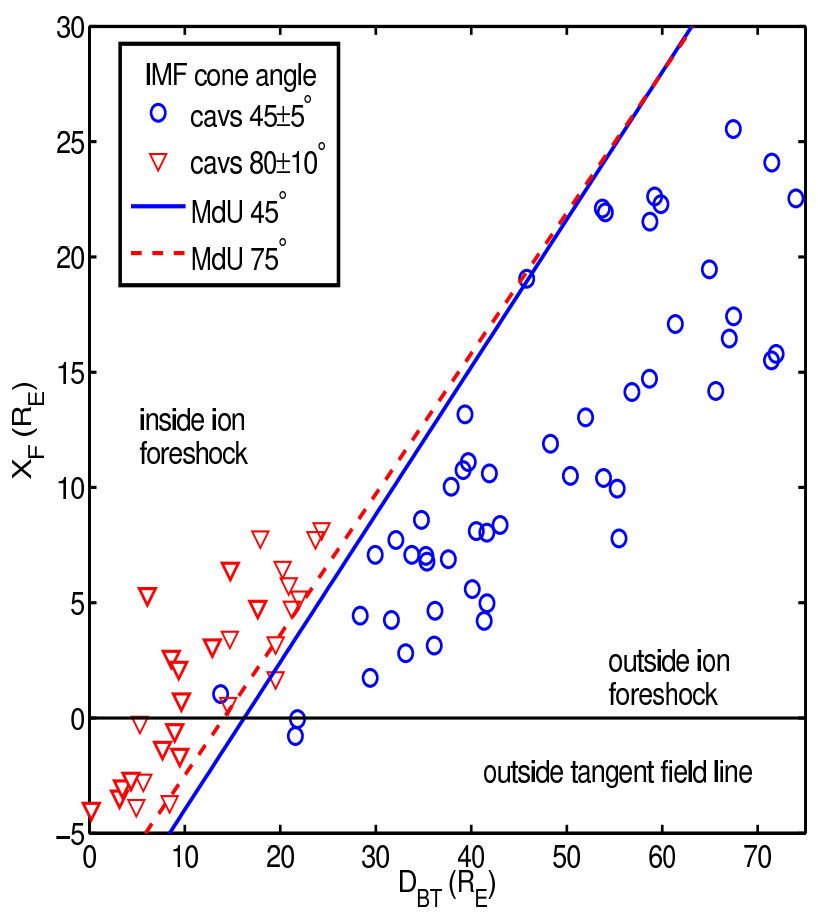

Fig. 6. SFC scatter plot of foreshock cavities observed for IMF cone angles $80^{\circ} \pm 10^{\circ}$ (red triangles) and those observed for IMF cone angles $45^{\circ} \pm 5^{\circ}$ (blue circles). The solid line indicates the intermediate ion foreshock boundary reported by Meziane and d'Uston (1998) for cone angles of $45^{\circ} \pm 5^{\circ}$, and the dashed line the same boundary for cone angles of $75^{\circ} \pm 5^{\circ}$. The boundaries reported by Meziane and d'Uston (1998) for cone angles of $75^{\circ}$ and $85^{\circ}$ are not significantly different (the gradients and intercepts of the linear fits are the same to within the errors reported). As relatively few foreshock cavities are observed for high cone angles, we employ wider bins.

Figure 7 illustrates how boundaries in the SFC system would map back to the more familiar GSE coordinates under the assumption of the magnetic field and the observation point being in the ecliptic plane. A schematic of the bow shock is drawn for two idealised upstream solar wind conditions. Blue/cyan lines correspond to an IMF cone angle of $45^{\circ}$ and red/magenta lines to $80^{\circ}$, the solid lines tangent to the shock surface show the tangent field line. In both cases the solar wind bulk flow velocity and magnetic field are, by construction, in the xy GSE plane. Dashed lines correspond to the intermediate ion foreshock boundaries of Meziane and d'Uston (1998). Cyan and magenta lines are the fits to our foreshock cavity observations, they become thicker to indicate the ranges over which cavities were actually observed. In the $\theta_{B X}=45^{\circ}$ case, cavities are observed near to and duskward of the tangent line, but well separated from intermediate ion foreshock line and sunward of it. At $80^{\circ}$ cone angle, the intermediate foreshock and cavities are less well separated, and cavities are observed both sides of the tangent line. Some cavities are observed around $5 R_{E}$ upstream of 


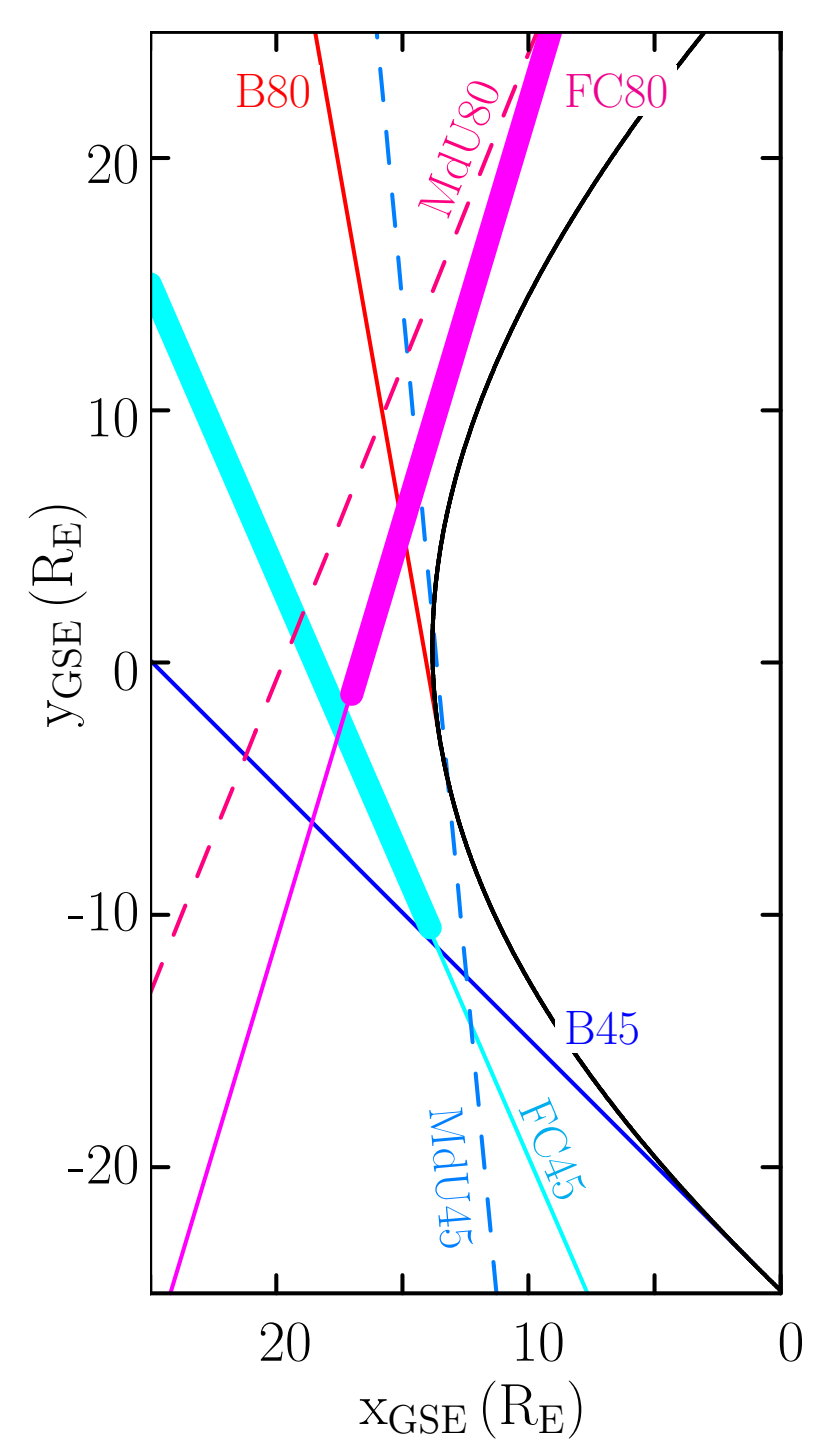

Fig. 7. An illustration of how the boundaries found in Solar Foreshock Coordinates (see Fig. 6) would map to the Geocentric Solar Ecliptic system if all observations and the solar wind magnetic field were constrained to the ecliptic plane. This schematic shows a section through the bow shock in the xy GSE plane for high and low IMF cone angles (by construction this plane contains the solar wind bulk flow velocity and the IMF). Solid blue and red lines indicate the position of the tangent field line for IMF cone angles of $45^{\circ}$ and $80^{\circ}$ (labelled B45 and B80). Dashed lines (MdU45 and MdU80) show the fits, reported by Meziane and d'Uston (1998), to the intermediate ion foreshock boundary. Magenta/cyan lines (FC45 and FC80) show linear fits to the locations of observed foreshock cavities; these lines thicken to indicate the regions in which cavities were actually found.

the point where the tangent field intersects the shock. If these cavities are transient encounters with the foreshock edge, the bow shock must somehow be displaced $\sim 5 R_{E}$ from its nominal position. The systematic change in cavity location with cone angle is, again (see Fig. 6) apparent; at $\theta_{B X}=45^{\circ}$ cavities are observed sunward of the nominal intermediate foreshock, but at $\theta_{B X}=80^{\circ}$ they are seen anti-sunward of it.

For cone angles of $45^{\circ}$ foreshock cavities are not observed collocated with the intermediate foreshock edge. However, the cavity observations lie between the intermediate ion foreshock and the tangent line. This suggests foreshock cavities may be transient encounters with the Field Aligned Beam foreshock region. Lines of constant energy (such as $W(\mathcal{E})$ in Fig. 4) are straight lines in Solar Foreshock Coordinates. The gradient of a line in SFC corresponds to a particular energy at a given cone angle:

$$
\frac{D_{B T}}{X_{F}}=\frac{v_{\| \text {beam }}}{v_{S W}}+\cos \theta_{B X}
$$

where $D_{B T}$ and $X_{F}$ are the abscissa and ordinate in SFC, $v_{\| \text {beam }}$ is the beam speed parallel to the magnetic field, $v_{S W}$ is in incident solar wind speed, and $\theta_{B X}$ is the IMF cone angle. For the foreshock cavities observed at $\theta_{B X}=45^{\circ}$, $v_{\| \text {beam }} \approx 1.8 v_{S W}$, this moderate energy is consistent with the $\sim 1 \mathrm{keV} \mathrm{e}^{-1}$ beam energy typical of a field aligned beam (e.g. Fuselier, 1994). However, this energy is small compared to the $\geq 27 \mathrm{keV}$ ions observed by RAPID inside each foreshock cavity. Additionally, the the line in Fig. 7 for cavities with $\theta_{B X}=45^{\circ}$, although downstream of the tangent field line, does not intersect the nominal bow shock surface. Foreshock cavities therefore cannot correspond to steady-state field aligned beams.

Foreshock cavities at typical $45^{\circ}$ cone angles are not found colocated with the intermediate ion foreshock edge, although the gradient of their locations in Solar Foreshock Coordinates can be associated with a modest constant beam speed. These cavities do not appear to be Field Aligned Beams of ions simply reflected from the bow shock. There is a systematic variation in foreshock cavity location with IMF cone angle. At low cone angles events are found downstream of the nominal intermediate ion foreshock whilst cavities observed at high cone angles are upstream of that boundary; a significant fraction of high cone angle cavities are upstream of the tangent field line.

\section{Cavity surroundings}

\subsection{Comparison to average solar wind}

Our next step is to compare the solar wind in which foreshock cavities are embedded to the ambient solar wind observed by Cluster during the survey period. The distributions of ambient IMF field strength, thermal ion density, bulk flow speed, and Alfvén Mach number for each of our 204 foreshock cavities are presented in the top row of Fig. 8. The first row shows that cavities occur in solar wind with moderate field strengths and low densities. The velocity distribution is almost flat between $300-700 \mathrm{~km} \mathrm{~s}^{-1}$. 


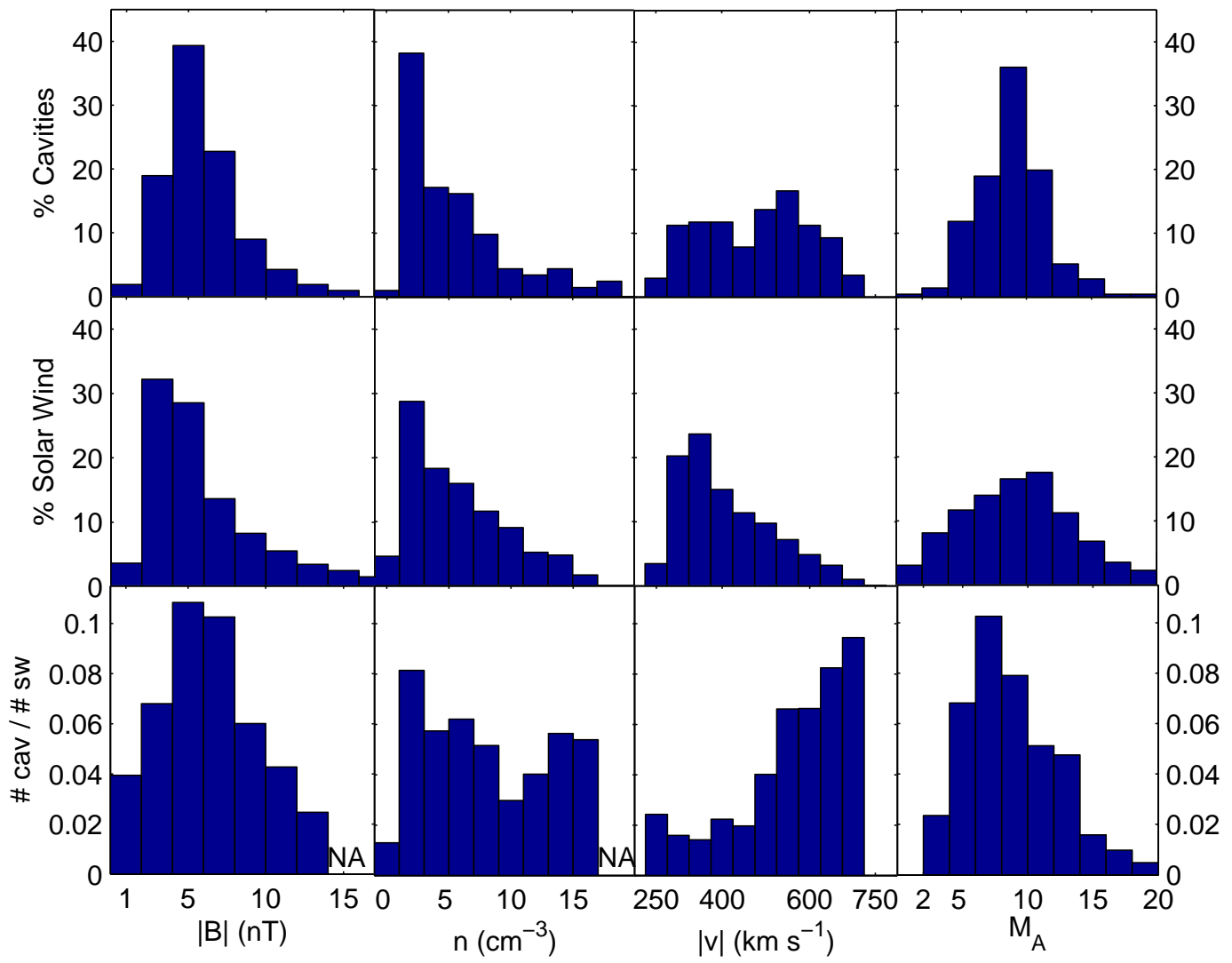

Fig. 8. Distributions of (in columns left to right): magnetic field magnitude, thermal ion density, bulk flow speed, and Alfvén Mach number, for (rows top to bottom): the foreshock cavities surveyed in this paper, hourly averages of solar wind during the survey period, occurrence patterns for the cavities normalised by those for the solar wind parameters. Hourly averaging for solar wind parameters suppresses the tails of their distributions in comparison to the distributions of the $\sim$ two-minute averages taken of cavity surroundings. This can lead to very high bin occupancy in the tails of the distributions when taking ratios. Affected bins have been suppressed to better illustrate the main results. Deleted bins are marked NA in the bottom row.

For comparison, the middle row of Fig. 8 plots histograms of hourly averages of the same parameters for the whole of Cluster dayside seasons 2005 and 2006. The magnetic field strength and density distributions generally resemble those for foreshock cavities. The bulk flow speed distribution for the hourly averages indicates that Cluster mostly encounters rather slow solar wind, with the number of hours spent measuring a given bulk flow dropping off rapidly for speeds faster than $400 \mathrm{~km} \mathrm{~s}^{-1}$. The distribution is not bimodal as might be expected from a simple picture of separate fast and slow solar wind streams.

The bottom row of Fig. 8 shows the number of foreshock cavities per bin, normalised by the number of hourly averages in that same bin. These normalised occurrence patterns indicate some preference for cavities to occur in moderate magnetic field strength solar wind streams. The low density peak is greatly reduced by normalisation, leaving the density occurrence distribution almost flat. The most striking tendency is for foreshock cavities to occur preferentially in high-speed solar wind, although care must be taken in the interpretation of the highest speed bins as division by the small number of solar wind intervals observed in this high speed tail may exaggerate their occupancy levels.

The preference for cavity occurrence in high speed solar wind is not, however, reflected in cavity occurrence normalised for Alfvén Mach number, which shows a broad peak at relatively low Mach numbers.

In the local hybrid simulation of Thomas and Brecht (1988), an ion beam propagating against a model solar wind is found to excavate a cavity only for Alfvén Mach numbers above 2. Cavity occurrence normalised be solar wind Mach number drops markedly for $M_{A}<5$ as might be expected if there existed a threshold Mach number below which cavities could not form; however we do observe a single cavity embedded in solar wind having $M_{A}<2$, the threshold found by Thomas and Brecht (1988). In any case, as shown in the central panel of the right-hand column in the Fig. 8, Cluster rarely encounters solar wind with such low Mach numbers; 

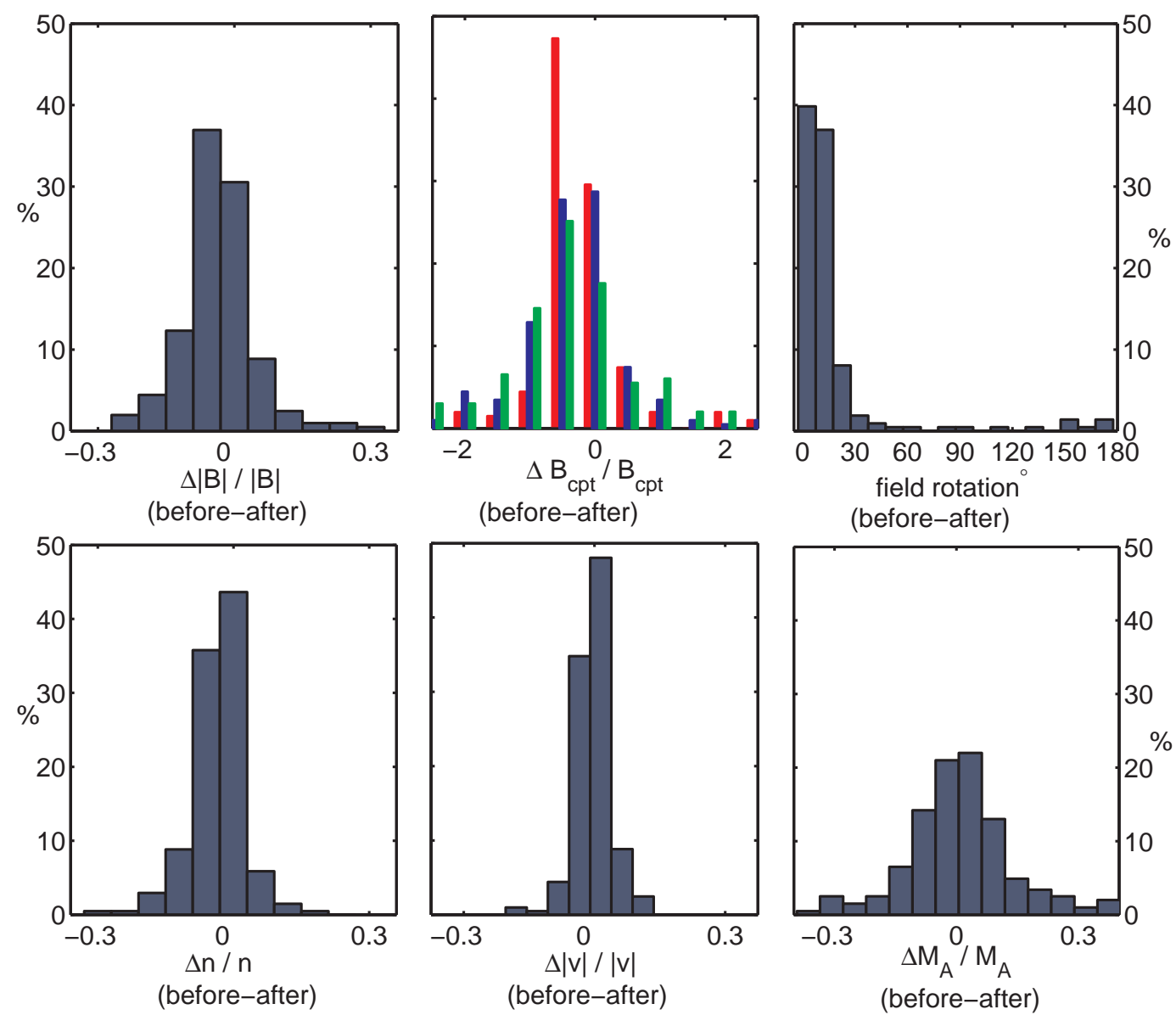

Fig. 9. Histograms of the differences between plasma conditions before and after observing foreshock cavities (clockwise from top left): magnetic field magnitude, GSE field components, field direction, Alfvén Mach number, CIS bulk flow speed, and CIS thermal ion density. Apart from field rotation and components, changes in each parameter have been normalised by the ambient values.

and this region of parameter space is not well explored. The tendency for fast solar wind streams to be relatively low density (e.g. Bruno and Carbone, 2005; Schwenn, 1990) tends to mask the solar wind speed dependence of cavity occurrence in the Mach number data.

It has been suggested that foreshock cavity suprathermal particles may have been accelerated from the bow shock in accordance with the Fermi model, mirroring from scattering centres which are approaching one another. The efficiency of the process depends only on the approach speed of the scattering centres, so long as the bow shock is supercritical and ion reflection at the shock significant. If the downstream scattering centres are relatively static near the shock, and the upstream scatterers are solar wind inhomogeneities, the acceleration will be more efficient in higher speed solar wind (see e.g. Scholer, 1985). The Mach number dependence would be seen only in the form of a threshold above which the shock is supercritical.

\subsection{Change in plasma parameters across foreshock cavities}

Previous studies of individual foreshock cavities have reported that (unlike Hot Flow Anomalies) there need not be a significant discontinuity from one side of a cavity to the other (e.g. Sibeck et al., 2004; Schwartz et al., 2006). However, no detailed, quantitative study of the change in magnetic field direction (and other plasma properties) across a large set of foreshock cavities has been reported.

In this section we investigate the change in plasma properties across foreshock cavities. Figure 9 presents histograms of the differences between intervals immediately before and after each foreshock cavity; all except the field rotation and components have been normalised to the greater of two measured values. The field rotation, top right panel, was tested against a null hypothesis. A zero-degree-mean gaussian distribution, with variance equivalent to measuring a $5 \mathrm{nT}$ field to $\pm 0.5 \mathrm{nT}$ precision, was "folded" around zero. No statistically significant difference was found in the field directions 
measured immediately before and after foreshock cavities. Similarly, there is no significant difference between the distribution of the change in field magnitude over foreshock cavities and a null hypothesis distribution based on measuring the same underlying field strength with small fluctuations superposed on it. The change in magnetic field over foreshock cavities fails to satisfy conventional criteria for identifying discontinuities (e.g. $|\Delta \boldsymbol{B}| /|\boldsymbol{B}| \geq 0.5$; see Tsurutani and Smith, 1979; Neugebauer and Alexander, 1991; Neugebauer et al., 1984). Unlike Hot Flow Anomalies, foreshock cavities do not form in association with global solar wind discontinuities.

Histograms of the changes in bulk plasma density and speed from before to after cavities are plotted in the bottomleft and bottom-centre panels of Fig. 9. Null hypothesis distributions were constructed based on the differences between two samples taken of the same plasma with a small statistical noise. The density and flow speed changes over foreshock cavities were found not to be statistically significant.

There are clearly discontinuities between the plasma in which a foreshock cavity is embedded and the cavity interior; most obviously in the magnetic field data. However, the field and thermal ion parameters return, after a cavity encounter, to essentially their pre-event values. Therefore we conclude that, unlike HFAs, foreshock cavities are not excavated along a global boundary between two distinct plasma populations.

\section{Cavity interiors}

We define the duration of a foreshock cavity as the time between spacecraft encounters with each edge of the magnetic field depression; a histogram of these durations is presented in Fig. 10. Although, some events exceed four minutes, durations of 90-180 s are more typical. These values are consistent with those, timed between the bounding magnetic field strength increases, reported by Sibeck et al. (2001). Such foreshock cavity durations correspond to a typical width of $\sim 8 R_{E}$ (assuming the spacecraft traverses the centre of a cavity which is convecting in the solar wind). This, at $\sim 200 c / \omega_{p i}$, is around three times the width of the cavities in Thomas and Brecht (1988)'s cavity model, but is in good agreement with the $\sim 9 R_{E}$ width of the magnetic depletion region found in the Skadron et al. (1986) foreshock model. The width of the initial beam in these simulations, along with the time allowed for the events to evolve, control the width of the depletion regions presented.

Next, we investigate the differences between the plasma parameters observed inside foreshock cavities and those in the surrounding solar wind. Figure 11 presents histograms of the differences, in the magnetic field and the thermal ion population, between the interiors of foreshock cavities and the exterior solar wind plasma within which they are embedded. Parameters other than field rotation and field component changes have been normalised by the average values

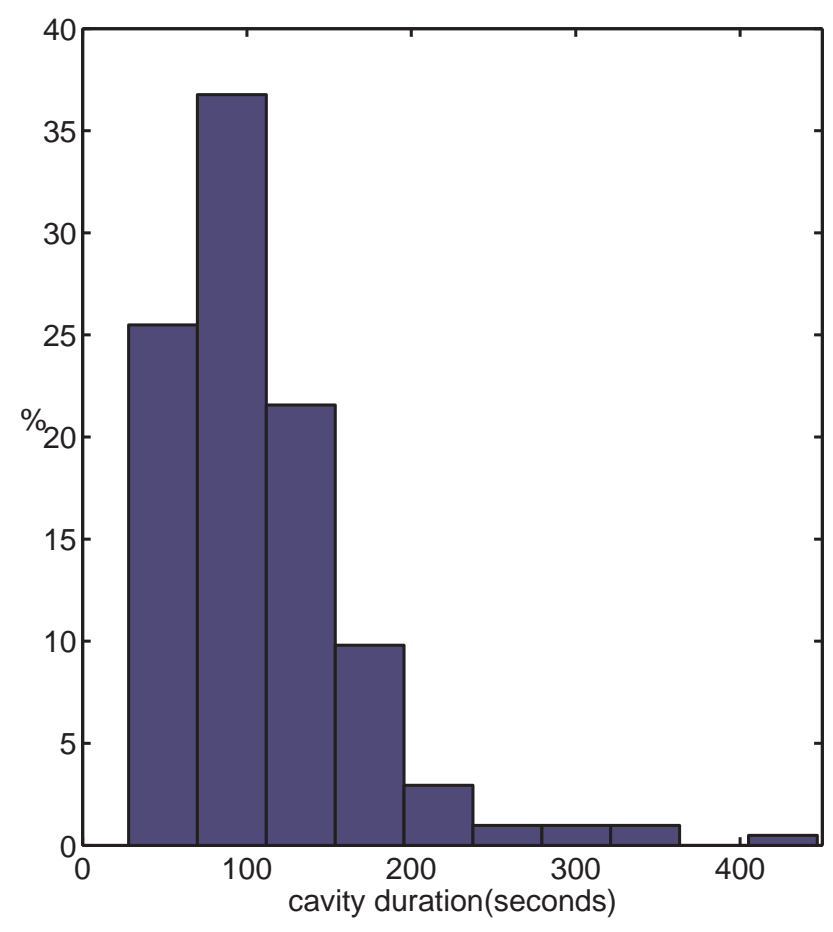

Fig. 10. Histogram of the durations of foreshock cavities. Timings were taken from the edges of the magnetic field depressions.

on either side of each cavity. The depressed magnetic field strengths and densities characteristic of cavity interiors can be seen in the top-left and bottom-left panels. On average, the magnetic field inside a cavity is $2.1 \mathrm{nT}$ lower than that outside, and the density decreases by $2.4 \mathrm{~cm}^{-3}$. For both parameters, these decreases correspond to interior values $\sim 0.6$ of those found outside; this value agrees well with the normalised cavity depth in the Thomas and Brecht (1988) simulation. Given the decrease in cavity depth with distance from the bow shock reported by Sibeck et al. (2004), our results are consistent with interior field strengths being $70 \%$ to $90 \%$ of ambient for the cavities seen by Sibeck et al. (2001) at the IMP-8 orbit. However the field variations we observe do not quite reach the factor of $\sim 3$ reported by some previous case studies (e.g. Sibeck et al., 1989).

The top right panel of Fig. 11 shows the distribution of the angle between the magnetic field observed inside foreshock cavities and the surrounding field. The average field changes direction by $45^{\circ}$, but the distribution is skewed toward larger rotations and has a large variance as a consequence of the noisy conditions typical inside foreshock cavities. The upper central panel of Fig. 11 shows the change in each of the components of the magnetic field in GSE coordinates. The distributions are similar in each direction, nearly symmetric about a peak near zero. Although there is significant change in the field direction from outside to inside most cavities, the change does not appear to occur with any preferred sense/direction. 

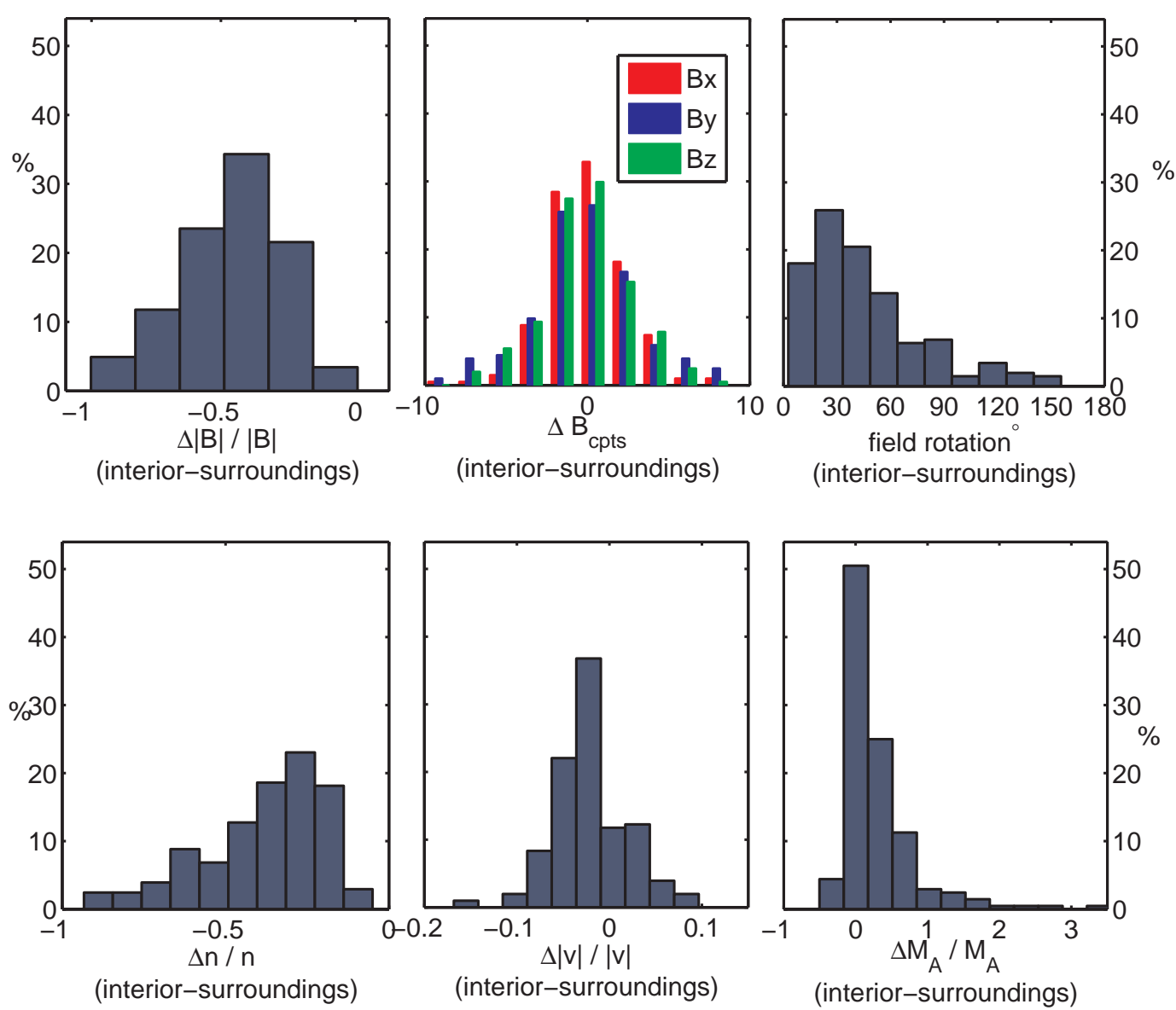

(interior-surroundings)

Fig. 11. Histograms of the differences between foreshock cavity centres and the surrounding plasma conditions in (clockwise from top left): magnetic field magnitude, GSE field components, field direction, Alfvén Mach number, CIS bulk flow speed, and CIS thermal ion density. Apart from field rotation and components, changes in each parameter have been normalised by the ambient values.

The lower centre panel of Fig. 11 presents a histogram of the differences in bulk flow speed between the interiors and environs of foreshock cavities. Flow speed may either increase or decrease with a slight bias in favour of slower values inside cavities. In all but 3 cases, deviations are less than $10 \%$ of the ambient solar wind speed; events with a greater than $20 \%$ speed change were excluded from the survey as probable HFAs.

The distribution of the change in Alfvén Mach number is shown in the lower right panel of Fig. 11. It is peaked near zero, with a substantial tail to the side of increasing Mach number: the magnetic field depletion dominates over the density decrease and any change in flow speed. However a small number of cavity interiors are at lower Mach numbers than their surroundings.

In summary the interiors of foreshock cavities have magnetic fields and thermal ion densities that are $\sim 60 \%$ of those outside, the depletions move over the spacecraft in $\sim 100 \mathrm{~s}$. The interior magnetic field makes a large (typically $45^{\circ}$ ) an- gle to the surrounding field. The bulk flow speed of the thermal ion population tends to be a little slower than that in the ambient solar wind; however this speed perturbation is much less than that found in Hot Flow Anomalies.

\section{Discussion}

If foreshock cavities were encounters with a boundary of the global foreshock, their locations should be scattered around the mean location of that boundary. Cavity observations at low and high cone angles are statistically separated from the nominal intermediate foreshock edge. Linear fits to locations of these cavities do not intersect the nominal bow shock surface as would be expected for transient encounters with the field aligned beam region.

We observe cavities in systematically different locations depending on the cone angle between the IMF and the EarthSun line. At low cone angles, cavities are found embedded in plasma upstream of the intermediate ion/ULF foreshock. As 
cavities can only be distinguished against a relatively quiescent background, we expect to preferentially select upstream events. However, cavities observed at high cone angles are located downstream of the nominal boundary of the intermediate ion foreshock. Yet, these cavities, in order to be identified, must have been embedded within relatively quiescent plasma; not the highly disturbed conditions expected downstream of the intermediate ion foreshock. There may be a selection effect due to the Cluster orbit being shock grazing, whilst the orbit of the IMP-8 spacecraft used for the intermediate ion foreshock survey was much further from the shock. There may also be some geometric effect due to the limited spatial extent of the global foreshock at high cone angles, when compared to the low cone angle foreshock.

Transient connection to the bow shock by an isolated ("rogue") bundle of magnetic flux, which points in a different direction to the field in the plasma surrounding it, is the simplest way to explain foreshock cavities seen upstream of the foreshock. These events cannot easily be explained by brief encounters with the global foreshock as it advances and retreats. However, we cannot conclusively rule out this possibility for all cavities, especially those observed at low cone angles. The low cone angle events are observed to occur well separated from the nominal intermediate ion foreshock edge, and they do not appear to be simply encounters with field aligned beams as fits to their locations do not intersect the nominal bow shock surface.

We observe few cavities at low Alfvén Mach numbers. and only a single event occurs below the $M_{A}=2$ threshold found in the simulations of Thomas and Brecht (1988). However, Cluster does not encounter low Mach number solar wind sufficiently often for us to draw any firm conclusions.

\section{Conclusions}

We have presented the results of a survey of foreshock cavities observed upstream of, but proximal to, the Earth's bow shock. These results, in the main, serve to confirm previously reported work on individual foreshock cavities and, the findings of a previous survey. The events are seen throughout those parts of the Cluster orbit that lay outside the shock. Cavities typically have durations in the range of $90 \mathrm{~s}$ to three minutes. The central density and magnetic field strength are depleted to around half of their ambient values. These values agree with the results of the Cluster case study of Schwartz et al. (2006) and with the previous results of Sibeck et al. (2001) and Sibeck et al. (2004) who found weak foreshock cavities in observations taken farther upstream of the bow shock and that event amplitude decreased with radial distance from the shock.

We find a marked tendency for foreshock cavities to occur in high speed solar wind; this finding confirms the result of Sibeck et al. (2001). There is also evidence of preferential cavity occurrence in solar wind streams with low den- sities and moderate magnetic field strengths. Solar wind streams with such parameters are generally associated with wind originating from coronal holes.

The finding from individual events that there need not be a large magnetic field or velocity shear from before to after the passage of a foreshock cavity is confirmed statistically. Indeed the average change in plasma parameters over a cavity is at the level of the differences between two measurements of pristine solar wind taken minutes apart.

Several questions about the origin and nature of foreshock cavities remain to be answered. With no obvious triggering event (for instance the interplanetary tangential discontinuities that generate Hot Flow Anomalies), it is difficult to estimate the total occurrence rate of foreshock cavities and hence, their aggregate impact on the dynamics of the magnetosphere.

We have not been able to definitively discriminate between the model of cavity formation on a isolated shock connected bundle of solar wind magnetic field and the description of cavities as transient encounters with the moving contiguous foreshock. It would, however, be difficult for the transient encounter model to reproduce the cavity locations we observe at high IMF cone angles.

Presently, there is no convincing explanation for the change in cavity location (with respect to the intermediate ion foreshock) with cone angle. Case studies should focus on exposing, in detail, the context of a foreshock cavity, and examining its position with respect to local foreshock encounters and bow shock crossings. Multi-spacecraft (perhaps multi-mission) studies should be able to employ an upstream monitor close that remains close to the site of a foreshock cavity without actually observing it and will then be able to observe any pre-existing 'rogue' field bundle in the upstream solar wind.

Acknowledgements. The authors would like to thank the FGM (PIs: A. Balogh/E. Lucek), and CIS (PIs: H. Rème/I. Dandouras), and RAPID (PI: P. Daly) instrument teams, and the teams responsible for the Queen Mary Science Analysis System, and the Cluster Science Data System. We acknowledge the support of the Royal Astronomical Society and the UK Institute of Physics C R Barber fund. This work is supported by UK STFC through the award of a studentship to LB and research grants to Imperial College London.

Topical Editor R. Nakamura thanks K. Meziane, G. Paschmann, and another anonymous referee for their help in evaluating this paper.

\section{References}

Balogh, A., Carr, C. M., Acuña, M. H., et al.: The Cluster Magnetic Field Investigation: overview of in-flight performance and initial results, Ann. Geophys., 19, 1207-1217, 2001, http://www.ann-geophys.net/19/1207/2001/.

Bruno, R. and Carbone, V.: The Solar Wind as a Turbulence Laboratory, Living Reviews in Solar Physics, 2, http://www. livingreviews.org/lrsp-2005-4, 2005. 
Eastwood, J. P., Lucek, E. A., Mazelle, C., Meziane, K., Narita, Y., Pickett, J., and Treumann, R. A.: The Foreshock, Space Sci. Rev., 118, 41-94, doi:10.1007/s11214-005-3824-3, 2005.

Edmiston, J. P., Kennel, C. F., and Eichler, D.: Escape of heated ions upstream of quasi-parallel shocks, Geophys. Res. Lett., 9, 531-534, 1982.

Fairfield, D. H.: Bow shock associated waves observed in the far upstream interplanetary medium., J. Geophys. Res., 74, 35413553, 1969.

Fairfield, D. H., Baumjohann, W., Paschmann, G., Luehr, H., and Sibeck, D. G.: Upstream pressure variations associated with the bow shock and their effects on the magnetosphere, J. Geophys. Res., 95, 3773-3786, 1990.

Fuselier, S. A.: Suprathermal Ions Upstream and Downstream From the Earth's Bow Shock, in: Solar Wind Sources of Magnetospheric Ultra-Low-Frequency Waves, edited by: Engebretson, M. J., Takahashi, K., and Scholer, M., 107 pp., 1994.

Fuselier, S. A., Thomsen, M. F., Gosling, J. T., Bame, S. J., and Russell, C. T.: Gyrating and intermediate ion distributions upstream from the earth's bow shock, J. Geophys. Res., 91, 91-99, 1986.

Greenstadt, E. W. and Baum, L. W.: Earth's compressional foreshock boundary revisited Observations by the ISEE 1 magnetometer, J. Geophys. Res. (Space Physics), 91, 9001-9006, 1986.

Greenstadt, E. W., Green, I. M., Inouye, G. T., Hundhausen, A. J., Bame, S. J., and Strong, I. B.: Correlated Magnetic Field and Plasma Observations of the Earth's Bow Shock, J. Geophys. Res., 73, 51-60, 1968.

Hoppe, M. M. and Russell, C. T.: Plasma rest frame frequencies and polarizations of the low-frequency upstream waves - ISEE 1 and 2 observations, J. Geophys. Res. (Space Physics), 88, 20212027, 1983.

Masters, A., Arridge, C. S., Dougherty, M. K., Bertucci, C., Billingham, L., Schwartz, S. J., Jackman, C. M., Bebesi, Z., Coates, A. J., and Thomsen, M. F.: Cassini encounters with hot flow anomaly-like phenomena at Saturn's bow shock, Geophys. Res. Lett., 35, 2202, doi:10.1029/2007GL032371, 2008.

Meziane, K. and d'Uston, C.: A statistical study of the upstream intermediate ion boundary in the Earth's foreshock, Ann. Geophys., 16, 125-133, 1998, http://www.ann-geophys.net/16/125/1998/.

Murr, D. L. and Hughes, W. J.: Solar wind drivers of Traveling Convection Vortices, Geophys. Res. Lett., 30, 1354, doi:10.1029/2002GL015498, 2003.

Neugebauer, M. and Alexander, C. J.: Shuffling foot points and magnetohydrodynamic discontinuities in the solar wind, J. Geophys. Res. (Space Physics), 96, 9409-9418, 1991.

Neugebauer, M., Clay, D. R., Goldstein, B. E., Tsurutani, B. T., and Zwickl, R. D.: A reexamination of rotational and tangential discontinuities in the solar wind, J. Geophys. Res. (Space Physics), 89, 5395-5408, 1984.

Øieroset, M., Mitchell, D. L., Phan, T. D., Lin, R. P., and Acuña, M. H.: Hot diamagnetic cavities upstream of the Martian bow shock, Geophys. Res. Lett., 28, 887-890, doi:10.1029/ 2000GL012289, 2001.

Paschmann, G., Haerendel, G., Sckopke, N., Moebius, E., and Luehr, H.: Three-dimensional plasma structures with anomalous flow directions near the earth's bow shock, J. Geophys. Res. (Space Physics), 93, 11 279-11 294, 1988.
Rème, H., Aoustin, C., Bosqued, J. M., et al.: First multispacecraft ion measurements in and near the Earth's magnetosphere with the identical Cluster ion spectrometry (CIS) experiment, Ann. Geophys., 19, 1303-1354, 2001, http://www.ann-geophys.net/19/1303/2001/.

Scholer, M.: Diffusive acceleration, in: Collisionless Shocks in the Heliosphere: Reviews of Current Research, edited by: Tsurutani, B. T. and Stone, R. G., vol. 35, pp. 287-301, Washington D.C. American Geophysical Union Geophysical Monograph Series, 1985.

Schwartz, S. J., Chaloner, C. P., Hall, D. S., Christiansen, P. J., and Johnstone, A. D.: An active current sheet in the solar wind, Nature, 318, 269-271, 1985.

Schwartz, S. J., Daly, P. W., and Fazakerley, A. N.: MultiSpacecraft Analysis of Plasma Kinetics, in: Analysis Methods for Multi-Spacecraft Data, edited by: Paschmann, G. and Daly, P. W., pp. 159-183, 2000a.

Schwartz, S. J., Paschmann, G., Sckopke, N., Bauer, T. M., Dunlop, M., Fazakerley, A. N., and Thomsen, M. F.: Conditions for the formation of hot flow anomalies at Earth's bow shock, J. Geophys. Res. (Space Physics), 105, 12 639-12650, doi: 10.1029/1999JA000320, 2000b.

Schwartz, S. J., Sibeck, D., Wilber, M., Meziane, K., and Horbury, T. S.: Kinetic aspects of foreshock cavities, Geophys. Res. Lett., 33, 12 103, doi:10.1029/2005GL025612, 2006.

Schwenn, R.: Large-Scale Structure of the Interplanetary Medium, Physics of the Inner Heliosphere I, 99 pp., 1990.

Sibeck, D., Kudela, K., Mukai, T., Nemecek, Z., and Safrankova, J.: Radial dependence of foreshock cavities: a case study, Ann. Geophys., 22, 4143-4151, 2004,

http://www.ann-geophys.net/22/4143/2004/.

Sibeck, D. G., Baumjohann, W., Elphic, R. C., Fairfield, D. H., and Fennell, J. F.: The magnetospheric response to 8-minute period strong-amplitude upstream pressure variations, J. Geophys. Res. (Space Physics), 94, 2505-2519, 1989.

Sibeck, D. G., Kudela, K., Lepping, R. P., Lin, R., Nemecek, Z., Nozdrachev, M. N., Phan, T.-D., Prech, L., Safrankova, J., Singer, H., and Yermolaev, Y.: Magnetopause motion driven by interplanetary magnetic field variations, J. Geophys. Res. (Space Physics), 105, 25 155-25 170, doi:10.1029/ 2000JA900109, 2000.

Sibeck, D. G., Decker, R. B., Mitchell, D. G., Lazarus, A. J., Lepping, R. P., and Szabo, A.: Solar wind preconditioning in the flank foreshock: IMP 8 observations, J. Geophys. Res. (Space Physics), 106, 21 675-21 688, doi:10.1029/ 2000JA000417, 2001.

Sibeck, D. G., Phan, T.-D., Lin, R., Lepping, R. P., and Szabo, A.: Wind observations of foreshock cavities: A case study, J. Geophys. Res. (Space Physics), 107, 4-1, doi:10.1029/ 2001JA007539, 2002.

Sibeck, D. G., Omidi, N., Dandouras, I., and Lucek, E. A.: On the Edge of the Foreshock: Model-Data Comparisons, Ann. Geophys., 26, 1539-1544, 2008, http://www.ann-geophys.net/26/1539/2008/.

Skadron, G., Holdaway, R. D., and Scholer, M.: Perturbation of the solar wind in a model terrestrial foreshock, J. Geophys. Res., 91, 8798-8804, 1986.

Slavin, J. A. and Holzer, R. E.: Solar wind flow about the terrestrial planets. I - Modeling bow shock position and shape, J. Geophys. 
Res. (Space Physics), 86, 11 401-11 418, 1981.

Sonnerup, B. U. Ö.: Acceleration of particles reflected at a shock front., J. Geophys. Res., 74, 1301-1304, 1969.

Thomas, V. A. and Brecht, S. H.: Evolution of diamagnetic cavities in the solar wind, J. Geophys. Res., 93, 11 341-11 353, 1988.

Thomsen, M. F., Gosling, J. T., Fuselier, S. A., Bame, S. J., and Russell, C. T.: Hot, diamagnetic cavities upstream from the earth's bow shock, J. Geophys. Res. (Space Physics), 91, 2961-2973, 1986.

Tsurutani, B. T. and Smith, E. J.: Interplanetary discontinuities Temporal variations and the radial gradient from 1 to $8.5 \mathrm{AU}, \mathrm{J}$. Geophys. Res. (Space Physics), 84, 2773-2787, 1979.
Wibberenz, G., Fischer, H. M., Zoellich, F., and Keppler, E.: Dynamics of intense upstream ion events, J. Geophys. Res., 90, 283-301, 1985.

Wilken, B., Daly, P. W., Mall, U., et al.: First results from the RAPID imaging energetic particle spectrometer on board Cluster, Ann. Geophys., 19, 1355-1366, 2001, http://www.ann-geophys.net/19/1355/2001/. 